\title{
Research and Development of PCB for Switching Power Supply
}

\author{
Wenjie Zhang ${ }^{1, \mathrm{a}^{*}}$, Jinfeng $\mathrm{Li}^{1, \mathrm{~b}}$ and Renbo $\mathrm{Xu}^{1,2, \mathrm{c}}$ \\ ${ }^{1}$ Nanchang Institute of Science and Technology, Nanchang, 330108, China \\ ${ }^{2}$ Physics and Microelectronics Institute, Central South University, Changsha 410083 china. \\ a568740355@qq.com, b524892454@qq.com, ${ }^{\mathrm{c}} 1310079831 @ q q . c o m$
}

*the corresponding author

\section{Keywords: Switching power supply; Semiconductor lighting; PCB; Driver}

\begin{abstract}
As the power source of the electronic equipment, the switch power is developed in the direction of efficiency, high power factor and low cost, and the core component high voltage power switch device is the main factor affecting the efficiency and reliability of the switching power supply. Therefore, it has practical significance for the design of the power supply circuit and the research of high voltage power devices. China has made rapid progress in the development of power devices, but there is a big gap between domestic production technology and foreign advanced level. Most businesses still use micron level technology lines, products are mainly in the middle and low ends, and foreign devices have occupied a huge market share because of the advantages of excellent performance and independent property rights. Therefore, the research and development of new device structure and its control of process flow are of practical significance. This paper focuses on the characteristics and main parameters of semiconductor lighting, and the design of PCB.
\end{abstract}

\section{Research Status and Development Trend}

First, high frequency and high power: the volume of the switching power supply is inversely proportional to the square root of the working frequency. On the premise of the same power capacity, the switching frequency can be greatly reduced. PWM and PFM chips with frequencies up to $1 \mathrm{MHz}$ have been developed abroad. The Power Integrations Inc in the United States is the first in the world to develop a successful three terminal isolated PWM type and turn off the power supply. After that, TOPSwitch, TOPSwitch-II, TOPSwitch-Fx, TOPSwitch-GX, PeakSwitch, LinkSwitch and other series of products have been introduced. PAM in China has successfully developed a high-power power supply with a single power of $48 \mathrm{~kW}$ and has been successfully applied to the electroplating industry by using full bridge phase shifted zero voltage switching technology. Using multiple modules parallel technology, Li Jinzhi successfully developed a high power switching power supply with a whole power of $150 \mathrm{~kW}$, in which the power of a single module is also $45 \mathrm{~kW}$, and it is successfully applied to the electrolysis power supply.

Secondly, the efficiency is high and the performance is good: because the power of the switching power supply is "open" and "shut", the current and voltage waveforms in the opening and closing process are overlapped and the switching loss is produced, which can not be ignored. Therefore, under the trend of high frequency development, soft switching technology emerges as the times require. At present, it is mainly through resonance to create zero voltage or zero current to open and turn off the switch tube to reduce the overlap of current waveform and voltage waveform, thus effectively reducing the loss and current and voltage stress of the switch tube, making the efficiency of the switching power supply more effective. It is necessary to use less devices as possible to improve the integration. This method not only reduces the volume of the whole machine, but also facilitates the design and manufacture of the whole machine.

Again, high pressure and high reliability: the development of switch power has always been closely related to the development of semiconductor devices, high frequency realization, the need for corresponding high performance semiconductor devices. At present, a bipolar transistor is used as a switching power supply for a power tube. The switching frequency can reach dozens of $\mathrm{kHz}$, 
and the switching frequency of switching power using power MOSFET can reach hundreds of kHz. However, for high voltage and high frequency power devices, it is still a difficult point in the design. In order to reduce the power loss of the device itself, the smaller the resistance of the device is, the better the better. In order to increase the frequency, the parasitic capacitance of the power is also required as small as possible. The contradiction between the three components of voltage, resistance and parasitic capacitance requires the optimum design of each structure parameter of the power device, and the researchers are also devoted to the research and development of the voltage resistance technology of the power devices.

\section{Driving Characteristics and Main Parameters of Semiconductor Lighting}

High luminance semiconductor lighting has the characteristics of high efficiency, energy saving, environmental protection, long life and so on. It is recognized as the most valuable "green" light source in twenty-first Century. It will replace white light and fluorescent lamp and become the mainstream in the field of lighting. Semiconductor lighting is not only suitable for general lighting, but also widely used in other fields.

Characteristics of Semiconductor Lighting Drive. First, the luminous efficiency is high, the luminous efficiency of semiconductor illumination can be achieved, and the monochromatic property is good and the spectrum is narrow. Now people are striving to improve the luminous efficiency and have made great progress. Secondly, energy saving, semiconductor lighting is a device that converts electric energy into light energy. It has high efficiency and low power consumption. Under the same lighting effect, the power consumption of semiconductor lighting is only $1 / 2$ of fluorescent lamps and 1/10 of incandescent lamps. Again, the effective life span is long, the average life span of the semiconductor lighting can reach 120 thousand hours. It is a few times more than the ordinary traditional lighting. The 24 hour continuous work can be used for $6 \sim 10$ years, which can be used for a long time and reduce the average use cost. Fourth, the dimming is good, compared with the traditional light source, the semiconductor lighting dimming performance is very ideal, and the control is convenient. Changing the forward current can easily adjust the color and intensity, and make the combination of light and color more diversified. Fifth, environmental protection and safety, semiconductor lighting is a typical green light source, cold light source, all solid state luminescence, does not contain toxic and harmful substances. Sixth, fast response, semiconductor lighting has nanosecond response speed, digital control and color dynamic changes can be realized.

Table 1 Comparison of performance comparison between LED lamp and ordinary energy saving lamp

\begin{tabular}{|l|l|l|}
\hline Contrast & LED lamp & Ordinary energy saving lamp \\
\hline strobing & Yes & No \\
\hline light & Too bright & soft \\
\hline Angle of irradiation & limited $120^{\circ}$ & Unlimited $360^{\circ}$ \\
\hline brightness & High brightness in a narrow range & Brilliance of brightness \\
\hline
\end{tabular}

Compared with ordinary lighting, semiconductor lighting has many advantages, but compared with energy-saving lamps, there are some shortcomings. Table 1 is the performance comparison table for the LED lamp and the ordinary energy saving lamp.

Therefore, the advantages of LED energy saving lamp as indoor lighting may not be more obvious than that of ordinary energy saving lamp, but it is still very excellent when the lamps and lanterns, such as flashlight, desk lamp, lamp and so on, only need to be irradiated in a narrow angle.

Characteristics and Main Parameters of Semiconductor Lighting Drive. Semiconductor lighting drivers have electrical, optical and thermal properties. First, the electrical characteristics of semiconductor lighting are basically the same as those of ordinary diodes, and the core is PN junction. Only when the voltage is greater than the conduction voltage of PN junction can the current flow through the PN junction. Fig. 1 shows the electrical characteristics of semiconductor lighting. From Fig. 1, we can see that fluctuations in voltage can cause large changes in current. 


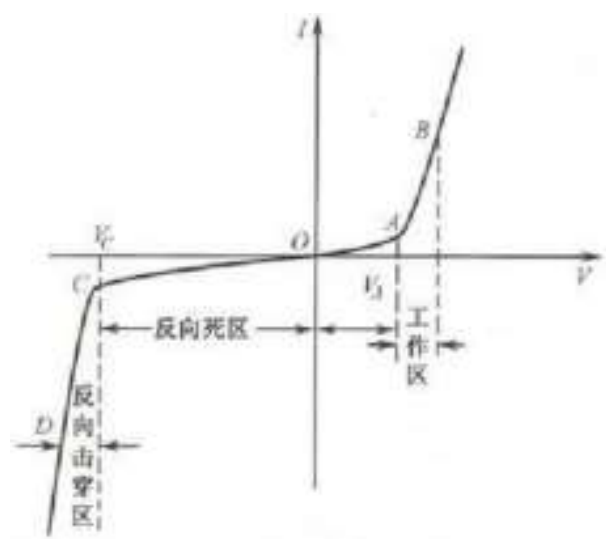

Figure 1. Electrical characteristics driven by semiconductor lighting

In Fig. 1, the A point is the opening voltage VT, the AB segment is the forward working area, the OC segment is the reverse characteristic area, and the CD segment is the breakdown area.

Secondly, the semiconductor lighting driver is illuminated by holes and electronic recombination. The more the number of composite carriers is, the stronger the luminescence is. The more carriers flow in PN junction within a unit time, the more the number of charges through the PN junction cross section, the greater the current flowing through the LED, and the luminance and the current flowing through the LED change positively. Fig. 2 is the relationship between luminous flux and LED current, that is, the optical characteristic curve.

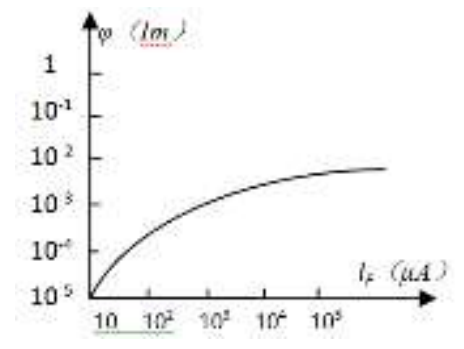

Figure 2. Optical characteristic curve of semiconductor lighting

Once more, when the temperature is greater than the critical value, the LED current decreases greatly. If the continuous increase of current will cause LED aging or burning, the good heat dissipation is the guarantee of the stable work of the LED. The curve of the temperature of semiconductor lighting with forward current is shown in Fig. 3.

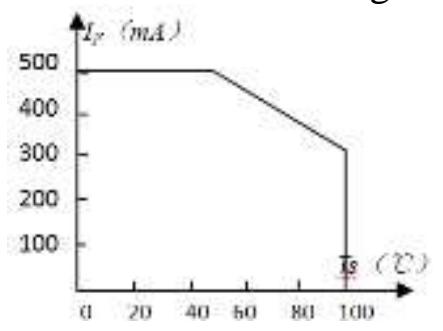

Figure 3. Thermal properties of semiconductor lighting

Fourth, semiconductor lighting has five main electrical parameters. The maximum forward current $I_{F m}$ is the maximum forward current that can be added at both ends of the semiconductor lighting. The maximum reverse voltage $V_{R m}$ is the maximum reverse voltage that can be added to both ends of the semiconductor lighting. The power consumption $P_{m}$ is allowed to be the maximum value of the positive voltage and current product allowed at both ends. The working environment temperature $T_{o p m}$ is the temperature range that the semiconductor lighting can work normally. 


\section{Design process of $\mathrm{PCB}$}

PCB Overall Design Process. PCB design is also an important part of switching power supply design, to prevent electromagnetic interference between and between wires and external radiation. Making the line connection in the most effective state, reducing the impedance of the line, and ultimately completing the finished product, the design flow of the switching power supply PCB is shown in Fig. 4.

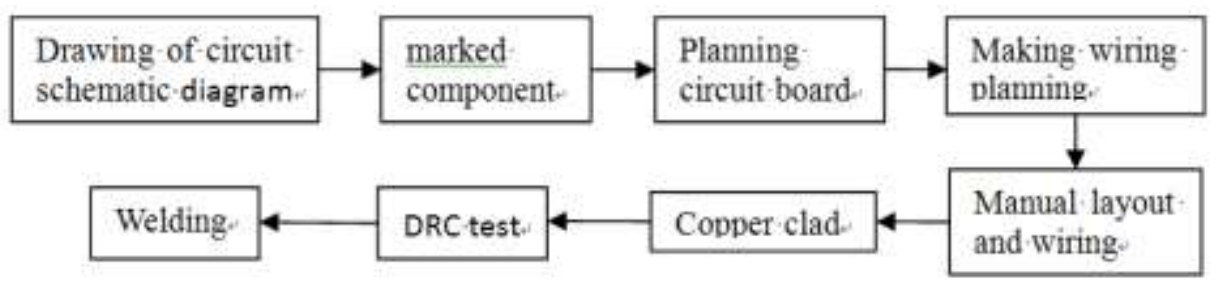

Figure 4. Design flow of switching power supply PCB

Layout Specifications of PCB. In order to make the PCB layout clear, it is necessary to modularized the PCB element device, that is to divide the module according to certain functions. In order to rationally layout the PCB structure, reduce the electromagnetic radiation of the inner and outer parts and optimize the main power circuit, the surrounding area should be reduced effectively; the arrangement direction of the PCB components should be in a certain order; the decoupling electricity is used. The capacity needs to be added to the power input of the integrated chip to provide a stable, less disturbing power supply; the switch's heat dissipation position should be installed reasonably and the heat sink position is placed on the side of the board and placed in the outside. In order to avoid the abnormal operation of the switch, the distance between the drive part of the control chip and the gate of the switch tube needs to be optimized. In general, the shorter the better.

Funded projects: Science and technology research project of Jiangxi Provincial Department of education in 2016. Project name: Application and research of dynamic reconfiguration in multiprocessor embedded system. Project number: GJJ161225.

\section{Summary}

As one of the most promising high-tech technologies in twenty-first Century, semiconductor lighting has entered a large scale LCD backlight system in the field of computer and smart TV from the original display, traffic signal, landscape lamp and other fields. It has been an important part of the "add glory" to the information age. Therefore, the research on semiconductor lighting has great social and practical significance. This paper will improve the stability of the driving control system, power factor, efficiency and anti-interference capability, and make a series of research and improvement, and the research work is limited. This paper focuses on the characteristics and main parameters of semiconductor lighting, the design method of PCB for switching power supply and so on. The work is very limited, and there are still many areas to be improved and perfected.

\section{Acknowledgements}

Project supported: Nanchang Key Laboratory of Applied Research of DME Optoelectronic Engineering (Nanchang Institute of Technology), No. NCZDSY -004.

\section{Reference}

[1] Sha Z.Y. Meng Z.Y. Ways to improve the efficiency of switching power supply. Power supply technologies and applications, 2012, 15(3): 60-64.

[2] Wang, X.B. The processing technology of High-frequency transformer in the switching power supply. The World of Power Supply, 2012:57-59. 
[3] Hazra, S. Bhattacharya, S. Chakraborty, C. A novel control principle for a high frequency transformer based multiport converter for integration of renewable energy sources. Industrial Electronics Society, IECON 2013-39th Annual Conference of the IEEE, 2013:7984-7989.

[4] F. C. Tien, C. H. Yeh, K. H. Hiesh. Automated visual inspection for microdrills in printed circuit board production. International Journal of Production Research, 2014, 42(12): 2477-2495.

[5] A. Kusiak, C.Kurasek. Data mining of printed-circuit board defects. IEEE Transactions on Robotics and Automation, 2011, 17(2):191-196.

[6] S. Oprea, I. Lita, I. B. Cioc. Determination of misplaced drill holes on a PCB. 30th International Spring Seminar on Electronics Technology, 2007, 7: 406-409.

[7] C. J. Chen, S. H. Lai, S. W. Liu, et al. Optical PCB inspection system based on Hausdorff distance. Machine Vision Applications in Industrial Inspection. 2015, 5679: 53-61.

[8] N. Talbot. The use of automated optical testing (AOT) in statistical process control (SPC) for printed circuit board (PCB) production. Circuit World, 2003, 29(4): 19-22.

[9] D. Craig. The Maturing PCB Industry. Printed Circuit Design and Manufacture, 2015,22(1):17-21.

[10]M. Gallant. Guide to Rigid-flex Design. Printed Circuit Design and Manufacture, 2016,23(10):30-33. 